\title{
Oral Health Behaviour of Qatari People Regarding Oral Cleaning Techniques
}

\author{
Alyafei Najat \\ Head of Preventative Oral Health Services, Primary Health Care Corp, Oral Health Division (Administration), Doha, Qatar
}

\begin{abstract}
Oral health behaviour varies according to the cultural norms, society, values and geographical region. Oral cleaning behaviour of the people living in Qatar predicts the prevalence rate of dental diseases that can be altered by oral cleaning and hygiene. The study comprised of a quantitative research study that is based on population sample of the nonprobability convenience sampling technique. The data collection has been conducted at Hamad Medical Corporation (HMC) where people were recruited and they were receiving dental appointments, as well as public and private dental clinic dental care. The primary outcomes of the study revealed that Qatari people need oral health education to prevent pain and disease. A weak association was found between the number of visits to the dental hygienist/dentist and gender. People with higher levels of education probably have the knowledge to obtain regular dental care from private practitioners and understand how a dental hygienist or dentist can benefit them. It could be concluded that this study has significantly represented the first known attempt to document the oral health behaviours of Qatari people regarding the cleaning techniques.
\end{abstract}

Keywords: Oral health; Oral hygiene; Dental; Dentistry

\section{Introduction}

Investigators throughout the world have proved that the oral health behaviour varies according to the cultural norms, values, and lifestyles [1]. Oral health behaviour refers to the response, attitude, and habits of the people regarding the hygiene, cleanliness and eating habits of the people [2]. The oral health behaviour of the people predicts the rate of prevalence of dental diseases which include gingivitis, periodontal disease, and periodontal or periapical abscesses [3]. Oral cleaning by the use of toothbrush and toothpaste, miswak, mouth cleansers or mouthwash refers to those techniques that are widely practiced throughout the world.

The Middle East has been heavily influenced by the traditional cleaning methods that are based on the miswak but now the tradition is rapidly changing and the majority of the Arab population now prefer to use the conventional method of a toothbrush with toothpaste [4]. Regarding the behaviour of the people living in Qatar, there is greater need to improve their knowledge regarding oral cleaning, dental treatments, and dental appointments so that the prevalence of dental diseases could be reduced in the region.

\section{The Significance of the Research}

The outcomes of this study are aimed to provide sufficient information regarding the oral health behaviour of the people living in Qatar so that the oral hygienists, dentists, and Qatari people may realize the importance of oral cleaning and hygiene that will ultimately result in decreasing the prevalence of dental diseases in Qatar.

Literature review: Different cultures and societies reflect a variety of norms, values, and preferences that differ among each other due to the differences in historical preferences, lifestyles, weather and many other constraints [5]. According to Anđelić et al. [6], there is a significant relationship between the lifestyle of the people and their health condition. Supporting the study, toothpaste Musaiger et al. [4], continued stating that oral health behaviour of the people is greatly influenced by the dietary habits, cultural norms, and lifestyle of the people.

Qatari people also show different behaviour regarding their health, treatment, and lifestyle. Honkala, has found that people living in Qatar are widely influenced by the lifestyles and preferences that running down from their ancestors [7]. Their behaviour is also reflected by the lifestyles that their Prophet Muhammad had adapted. Amin and AlAbad, have linked the oral health behaviour of Qatari people with the traditional oral cleaning technique among Muslims. The study stated that majority of the Qatari people prefer to use miswak only while some prefer to use both miswak and toothbrush with toothpaste [8-10].

Arab people have used various methods including herbs to treat oral pain and clean teeth. Al-Saqiley, Hanna, and Musketeer discussed the traditional Arab teeth cleaning methods, including fruits and herbs used to treat gingivitis and stomatitis. Al-Saqiley asserts that eating fish, sour milk, sesame, fenugreek, acidic foods like vinegar and lemon, sweets, and drinking very cold or hot beverages weaken gingiva and teeth in both children and adults. To treat gingivitis and stomatitis, Al- Saqiley recommended rinsing the mouth with water that had been used to cook lentils in order to reduce inflammation or irritation. Bent fleece soaked in hot oil (any oil), and applied to the gingiva is recommended to relieve pain. Hanna advocated the treatment of gingivitis in children and adults with a mouth rinse of water, salt, and thyme. The concentration of thyme should be twice the amount of salt in the mixture. The free gingiva also can be rubbed with the mix two to three times a day, and the rubbing should be done until the tissue bleeds. The foundation of this practice is the belief that bleeding indicates the removal of inflammation and ulcers. Gingival swelling in children also is treated by applying certain herbs, such as Hassan Yousef and Al-nelah; however, no research could be found to substantiate their effectiveness. While Hassan Yousef used on the gingiva to reduce gingivitis, Al-nelah and kalebo are mixed with water and applied to a baby's gingiva to reduce swelling associated with

${ }^{*}$ Corresponding author: Alyafei Najat, Head of Preventative Oral Health Services, Primary Health Care Corp, Oral Health Division (Administration), Izqawa, Alwakrah street Villa \#106, Doha, Qatar, Tel: +442032892633; E-mail: alyafeinajat@gmail.com

Received June 23, 2018; Accepted July 09, 2018; Published July 13, 2018

Citation: Najat A (2018) Oral Health Behaviour of Qatari People Regarding Oral Cleaning Techniques. Dentistry 8: 498. doi:10.4172/2161-1122.1000498

Copyright: (c) 2018 Najat A. This is an open-access article distributed under the terms of the Creative Commons Attribution License, which permits unrestricted use, distribution, and reproduction in any medium, provided the original author and source are credited. 
the eruption of primary teeth. Raisins are used for gingival swelling, as are pomegranate peels soaked in water [11]

\section{Methodology}

Since the research is aimed to explore the oral health behaviour of the Qatari people, therefore the study has been conducted in Doha, Qatar.

\section{Research Design}

The research is based on the quantitative analysis of the data that has been collected through various primary resources. The quantitative research design has enabled the study to be relevant, reliable and focused on the oral health behaviour of the Qatari people regarding oral health techniques.

\section{Data Collection Technique}

A self-designed questionnaire entitled Oral Health Behaviours of Qatari People was distributed among Qatari people in the State of Doha. Since Arabic is the official language in the country, both the questionnaire and the cover letter were translated. The questionnaire included three sections: the first section, on demographics, addressed age, gender, marital status, and educational level of the respondents. The second section addressed participants' oral health behaviours and included questions about their self-care behaviour. The third section surveyed participants' attitudes about the oral hygiene and cleaning techniques.

\section{Data sampling}

The nonprobability convenience sample $(\mathrm{n}=1000)$ included patients scheduled for a dental appointment in Hamad General CorporationDental Department and the Public Dental Health Care Centres and private dental clinics. Also included were students enrolled at the University of Qatar, and Governmental Schools, persons employed at the Police and Army Departments, and other public members. These individuals were accessible because of their scheduled dental, and/or dental hygiene appointments at public centres or because they were in other public locations. To be included, subjects had to be within 1872 years of age, and participation was not limited by health or dental history. Participation was voluntary and not obligatory. The final sample, $n=859$, was $41 \%$ male and $58.3 \%$ female. About $41 \%$ of the respondents were $18-25$ years of age, $30.5 \%$ were between $26-32 ; 14.9 \%$ were between $33-40 ; 7.7 \%$ were between $41-48 ; 3.3 \%$ were between 49-56; $1.2 \%$ was between $57-64$, and $1.0 \%$ was between $56-72$. Most of the respondents hold a baccalaureate degree (49.2\%), followed by high school graduates (29.6\%), elementary school graduates $(10.8 \%)$, individuals with graduate education (5.4\%), and individuals with no formal education $(5.0 \%)$.

\section{Ethical considerations}

To consider the ethical aspects of the study and the participants, a cover letter was attached to the questionnaire that clarified the aim of the research and explained how information from the participants will improve the health status and quality of life of Qatari people. The cover letter also informed the participants that the data will be kept confidential, and participants were informed that they could receive the results of the study by submitting their names and addresses to the principal investigator and/or the investigator assistants' after data collection

To value the Protection of Human Rights, a consent form was submitted to Old Dominion University Institutional Review Board for the Protection of Human Subjects; for which approval was received on March 9, 1998. The consent informed the Board that all the participants volunteered themselves to response the study questionnaire and they were free to withdraw from participating at any stage of the study.

\section{Risk and Limitations Factors}

No risks were involved in this study. Results will be used to develop programs and procedures to improve the oral health attitudes, knowledge, and behaviours of Qatari people. The limitations that were encountered in the study consist of the generalization of the findings to Qatari people who have characteristics similar to those found in the sample and people 18 years of age or lesser have not been represented in this study.

\section{Results}

Demographic information was obtained from items one through four located in section one of the questionnaire. Item one asked respondents to indicate their age from the following increments: 18-25, 26-32, 33-40, 41-48, 49-56, 57-64, and 56-72. Findings indicated that $41.4 \%$ of respondents were $18-25$ years of age $(n=356) ; 30.5 \%(n=262)$ were between $26-32 ; 14.9 \%(n=128)$ were between $33-40 ; 7.7 \%(n=66)$ were between $41-48 ; 3.3 \%(n=28)$ were between $49-56 ; 1.2 \%(n=10)$ were between $57-64$; and $1.0 \%(n=9)$ were between $56-72$.

Item two asked the respondents to indicate their gender. Responses indicated that $41.7 \%(n=358)$ of the respondents were male while $58.3 \%$ $(\mathrm{n}=501)$ of the respondents were female.

Item three asked respondents to indicate their highest level of education. Five percent $(n=43)$ of the respondents had no formal education; $10.8 \%(n=93)$ were elementary school graduates; $29.6 \%$ $(n=254)$ were people with high school diploma; $49.2 \%(n=423)$ were baccalaureate graduates; and $5.4 \%(n=46)$ of the respondents had graduate degrees.

To identify the oral health behaviours of Qatari people, item four provided information related to this question. Item 5 asked subjects to indicate if they ever visited a dental clinic to have their teeth cleaned. Responses from item 5 revealed that $74.4 \%(\mathrm{n}=639)$ of respondents received professional dental cleanings, while 25.6\% $(n=220)$ never received dental cleanings. Item 6 requested that respondents indicate if they ever received tooth brushing instructions at the dental clinic. Responses revealed that $37.8 \%(n=325)$ of the respondents had received tooth brushing instructions at the dental clinic and $62.2 \%(n=534)$ had not received any tooth brushing instructions.

Regarding the number of times respondents brush their teeth using a toothbrush, $2.6 \%$ do not brush their teeth $(n=22) ; 2.0 \%(n=17)$ brush their teeth on special occasions; $0.8 \%(n=7)$ brush their teeth once a month; 3.6\% $(n=31)$ brush their teeth once a week; $29.2 \%(n=251)$ brush their teeth once a day; and $61.8 \%(\mathrm{n}=531)$ brush their teeth more than once a day.

When considering the number of times Qatari people brush their teeth with a toothbrush, there was a statistically significant relationship with age $\left(x^{2}=69.687, d f=30, p=.000\right)$, gender $(\% 2=58.194, d f=5$, $\mathrm{p}=.000)$, and highest level of education $(\% 2=37.889, \mathrm{df}=20, \mathrm{p}=.009)$. There was no association between the number of times Qatari people brush their teeth with a toothbrush and distance from a dental clinic $\left(\mathrm{x}^{2}=10.202, \mathrm{df}=15, \mathrm{p}=.807\right)$.

Analysis of residuals revealed that 118 people in the $18-25$ age 
group and 5 from the 57-64 age group were more likely to brush their teeth once a day. Age groups 26-32 $(n=176)$ and 33-40 $(n=88)$ were more likely to brush their teeth more than once a day; the 41-48 $(n=8)$ age group was more likely to not brush their teeth. Furthermore, 25 of Qatari males were more likely to brush once a week, while 348 of the Qatari females were more likely to brush their teeth more than once a day.

Item 7 asked subjects to indicate the amount of time they spent each day cleaning their teeth. Responses revealed that $17.6 \%(n=151)$ of the respondents spent less than one minute each day cleaning their teeth; $41.7 \%(n=358)$ spent more than one minute but less than two minutes each day cleaning their teeth; $21.0 \%(n=180)$ spent more than two minutes but less than three minutes each day cleaning their teeth; $10.0 \%$ $(\mathrm{n}=86)$ spent more than three minutes but less than four minutes each day cleaning their teeth; $5.8 \%(n=50)$ spent more than four minutes but less than five minutes each day cleaning their teeth; and $4.0 \%(n=34)$ spent more than five minutes each day cleaning their teeth.

A statistically significant relationship was established between the period of time Qatari people spend each day cleaning their teeth and age $\left(x^{2}=47.892, d f=30, p=.020\right.$. Analysis of residuals revealed that Qatari people ages 18-25 ( $\mathrm{n}=73)$ were more likely to brush their teeth for less than one minute; persons within the 26-32 $(n=120)$ and 49-56 $(n=14)$ age groups were more likely to spend more than one minute but less than two minutes; 33 persons in the age group 33-40 and 6 persons in the age group 41-48 were likely to spend more than two minutes but less than three minutes brushing their teeth. No statistically significant association was found between the period of time Qatari people spent each day cleaning their teeth and the following demographic variables of gender $\left(\mathrm{x}^{2}=8.192, \mathrm{df}=5, \mathrm{p}=.146\right)$ and the highest level of education $\left(\mathrm{x}^{2}=24.399, \mathrm{df}=20, \mathrm{p}=225\right)$.

In item 9 subjects were asked to indicate when they cleaned their teeth: after waking up, after eating breakfast, after eating dinner, before going out, before going to bed, prior to special occasions, e.g., parties and holidays. Findings from item 14 revealed that $74 \%(n=641)$ of the respondents cleaned their teeth after waking up; $18.4 \%$ ( $n=158$ another item asked subjects to indicate the amount of time they spent each day cleaning their teeth. Responses revealed that $17.6 \%(n=151)$ of the respondents spent less than one minute each day cleaning their teeth; $41.7 \%(n=358)$ spent more than one minute but less than two minutes each day cleaning their teeth; $21.0 \%(\mathrm{n}=\mathrm{l} 80)$ spent more than two minutes but less than three minutes each day cleaning their teeth; $10.0 \%$ $(n=86)$ spent more than three minutes, but less than four minutes each day cleaning their teeth; $5.8 \%(n=50)$ spent more than four minutes but less than five minutes each day cleaning their teeth, and $4.0 \%(n=34)$ spent more than five minutes each day cleaning their teeth.

A statistically significant relationship was established between the period of time Qatari people spend each day cleaning their teeth and age $\left(\mathrm{x}^{2}=47.892, \mathrm{df}=30, \mathrm{p}=.020\right)$. Analysis of residuals revealed that Qatari people ages 18-25 ( $\mathrm{n}=73)$ were more likely to brush their teeth for less than one minute; persons within the 26-32 $(n=120)$ and 49-56 $(n=14)$ age groups were more likely to spend more than one minute but less than two minutes; 33 persons in the age group 33-40 and 6 persons in the age group 41-48 were likely to spend more than two minutes but less than three minutes brushing their teeth. No statistically significant association was found between the period of time Qatari people spent each day cleaning their teeth and the following demographic variables of gender $\left(\mathrm{x}^{2}=8.192, \mathrm{df}=5, \mathrm{p}=.146\right)$ and the highest level of education $\left(x^{2}=24.399, d f=20, p=225\right)$.

In the subsequent item, subjects were asked to indicate when they cleaned their teeth: after waking up, after eating breakfast, after eating dinner, before going out, before going to bed, prior to special occasions, e.g., parties and holidays. Findings from item 14 revealed that $74 \%$ $(n=641)$ of the respondents cleaned their teeth after waking up; $18.4 \%$ $(n=158)$.

\section{Discussion}

Qatari people visit dental clinics to have their teeth professionally cleaned; few dentists devote time to client education. Therefore, data suggest a need for oral health professionals who will invest treatment time in preventive oral health instructions. Al- kuwari mentioned in his dissertation that no dental hygienists exist in the State of Qatar and their role in education and preventive therapy could be an important contribution to the oral health of the people [9].

The number of Qatari people who have never been to a dental hygienist/dentist might be an underestimation since most of the people in the survey were waiting to be treated at a dental clinic. To improve their quality of life, Qatari people could benefit from to receive regular preventive care and should not wait until they experience pain before seeking professional treatment. These findings further suggest that Qatari people need oral health education to prevent pain and disease. These findings not only apply to Qatari people, but also to the Omanis, who share a similar culture, and who like the Qatari people, go to the dentist when they experience pain. About $54 \%$ of the Omanis population have never been to a dentist.

A weak association was found between the number of visits to the dental hygienist/dentist and gender. Residual analysis revealed that females were more likely to visit the dental hygienist/dentist than males when they experienced pain. Perhaps women were more likely than men to experience dental pain or preferred to visit the dental hygienist/ dentist for emergency only.

Data suggest that Qatari people surveyed with no formal education and elementary school education were more likely to avoid the dental hygienist/dentist, high school diplomas were more likely to visit dental hygienist/dentist for cosmetic reasons; while those with baccalaureate degrees were more likely to visit the dental hygienist/dentist once a year, and Qatari people with graduate degrees were less likely to visit the dental hygienist/dentist. Perhaps less educated Qatari people to adhere to the traditional philosophy that healthcare is sought only when necessary. People with higher levels of education probably have the knowledge to obtain regular dental care from private practitioners and understand how a dental hygienist or dentist can benefit them. People who are less educated tend to have external loci of control and would, therefore, perceive dental diseases in fatalistic terms. In contrast, persons with higher levels of education tend to have internal loci of control and therefore embrace preventive dental concepts as something they can do to thwart dental disease. In addition, people who are less educated may have experienced dental pain, extractions, and tooth loss at an early age, and therefore come to accept poor dental health, disease, and pain as a normal part of life.

An association was found between the number of times Qatari people brush their teeth and the variables of age, gender, and level of education. Data suggest that younger people are more likely to brush their teeth once or more a day, while age groups 41-48 are more likely to not brush their teeth at all. The younger Qatari people may be more modern, may have learned oral health concepts in schools or from television, and may have the willingness to accept new preventive measures. 
Majority of the Qatari people use charcoal and salt to clean their teeth. This finding is supported by Hanna (1989) who found that some Arab people use a mixture of ground charcoal with salt and water to clean their teeth. Of the respondents, $21.0 \%$ use miswak to clean their teeth, which vary significantly from the Jordanian population where the majority $(75 \%)$ use miswak to clean their teeth.

\section{Conclusion}

The Qatari family has been described as traditional, paternal, extended, endogamous, authoritarian, and occasionally polygamous. There is minimal or no dental hygienists or programs designed to train and graduate professional dental hygienists. Arab people have used various methods including miswak, toothbrush, and mouthwash, herbs to treat oral pain and clean teeth. Most Qatari people visit a dental clinic for professional dental cleaning as dental services are free for the citizens, but only a few of the people who visit a dental clinic show positive response by improving the oral cleaning practices. Majority of the Qatari people use a toothbrush and toothpaste to clean their teeth for more than once a day and spend an average of 2 minutes and 57 seconds cleaning their teeth each day. This study has significantly represented the first known attempt to document the oral health behaviours of Qatari people regarding the cleaning techniques.

\section{References}

1. Amin TT, Al-Abad BM (2008) Oral hygiene practices, dental knowledge, dietary habits and their relation to caries among male primary school children in Al Hassa, Saudi Arabia. Int J Dent Hyg 6: 361-370.
2. Cheema S, Maisonneuve P, Al-Thani MH, Al-Thani AAM, Abraham A, et al (2017) Oral health behaviour and factors associated with poor oral status in Qatar: results from a national health survey. J Public health dent 77: 308-316.

3. Al-Darwish MS (2016) Oral health knowledge, behaviour and practices among school children in Qatar. Dent Res J 13: 342.

4. Musaiger AO, Al-Mannai M, Abduljawad E (2014) Association of oral hygiene habits and food intake with the risk of dental caries among undergraduate university women in Saudi Arabia. Int J Adolesc Med Health 26: 585-589.

5. Al-Darwish MS, Abuhassna M, Al-Thomairy SA (2015) Oral Health Knowledge and Sources of Oral Health Information among School Children in Qatar. J Dent Health Oral Disord Ther 2: 00049.

6. Andelic I, Matijevic S, Andelic J (2015) The importance of oral health behaviour of children for their oral health. Sanamed 10: 101-107.

7. Honkala E (2014) Primary oral health care. Med Princ Pract 23(Suppl 1): 17-23.

8. Al-Darwish M, El Ansari W, Bener A (2014) Prevalence of dental caries among 12-14 year old children in Qatar. Saudi Dent J 26: 115-125.

9. AI-Salahi NAJS (1998) Oral health knowledge, attitudes, and behaviours of Qatari people.

10. Amin WM, AL-Omoush SA, Hattab FN (2001) Oral health status of workers exposed to acid fumes in phosphate and battery industries in Jordan. Int Dent J 51: 169-174.

11. Widiati S, Santosa AS, Prabandari YS, Prawitasari JE (2016) Oral health status of elementary-school children varied according to school they attended. Dental Journal (Majalah Kedokteran Gigi) 49: 163-167. 